\title{
CAN CLASSICAL DESCRIPTION OF PHYSICAL REALITY BE CONSIDERED COMPLETE?
}

\author{
GABRIEL CATREN \\ CREA, UMR 7656, ÉCOLE POLYTECHNIQUE/CNRS, 1 RUE DESCARTES, \\ 75005, PARIS, FRANCE

\begin{abstract}
We propose a definition of physical objects that aims to clarify some interpretational problems in quantum mechanics. We claim that the transformations induced by an objective property of a physical system must leave invariant all the other objective properties of the same system. The uncertainty principle is understood as a natural consequence of the imbrication between objective properties and non-objective properties. It follows from the proposed definition that in classical mechanics non-objective properties are wrongly considered objective. We conclude that, unlike classical mechanics, quantum mechanics provides a complete objective description of physical systems.
\end{abstract}

\section{INTRODUCTION}

According to Einstein, quantum mechanical description of physical reality cannot be considered complete. In his words, there are 'elements of physical reality' that do not 'have a counterpart in the physical theory'. ${ }^{1}$ In classical mechanics, the exact position and the exact momentum of a particle can be simultaneously predicted for all times from a given set of initial conditions. In quantum mechanics, on the other hand, the momentum of a system characterized by a well-defined position cannot be predicted by the theory (and vice versa). More generally, Heisenberg's uncertainty principle states that canonically conjugated variables can be simultaneously predicted up to some inversely

\footnotetext{
${ }^{1}$ This is the conclusion of the seminal Einstein-Podolsky-Rosen article (Einstein et al. [1935]). An historical account can be found in (Mittelsteadt [2006]).
} 
correlated uncertainties. The conceptual content of this principle has been the object of a heated debate that remains unresolved to this day. ${ }^{2}$

In this paper, we argue that quantum mechanics can be understood as the formalization of a rigorous definition of physical objects. According to the standard characterization, the objective properties that define a physical object are invariants under a certain set of transformations (Auyang [1995]; Born [1998]; Nozick [1998]; Weyl [1952]). However, there is no general prescription for determining which transformations are needed in order to define the objective properties of a given physical system. Our definition of physical objects claims that these transformations are induced by the objective properties themselves. In other words, we argue that the transformations induced by the objective properties of a physical system must be automorphisms of the system. This definition imposes a compatibility condition on the set of objective properties of a given object. This condition requires that an objective property be invariant under the transformations induced by the other objective properties of the same object. The significant result is that this compatibility condition is not consistent with classical mechanics, but rather with quantum mechanics.

According to our definition of physical objects, the uncertainty principle is the formal translation of the imbrication between objective properties and non-objective properties. As we shall see, asking which position is objective in a quantum system with a well-defined momentum is as nonsensical as asking which side of a die is the objective (or privileged) one. This means that in classical mechanics non-objective elements of physical reality are wrongly considered objective. On the other hand, we claim that quantum mechanics provides a complete description of all the objective properties of a physical object. It follows that the quantum description of a physical object is not incomplete,

\footnotetext{
${ }^{2}$ Many interpretations were proposed for the uncertainty principle. It was alternatively interpreted as a consequence of the unpredictable perturbations in experimental measures of physical quantities, as a result of the mutual incompatibility of certain experimental contexts, in terms of a subjective lack of knowledge of welldefined objective states, as a description of the statistical spread in an ensemble of similarly prepared systems, as the manifestation of an ontological indeterminateness in the definition of physical quantities, etc. (see for example Hilgevoord and Uffink [2006]).
} 
but rather that classical states are specified by means of too many variables. Since quantum states only describe all the objective properties of the object, they depend on half of the classical variables.

This article develops, in more conceptual terms, the interpretation of quantum mechanics begun in Catren [2008]. This interpretation is founded on an analysis of the symplectic formulation of mechanics (Abraham and Marsden [1978]; Libermann and Marle [1987]; Marsden and Ratiu [1999]; Souriau [1997]) and the geometric quantization formalism (Brylinski [1993]; Kostant [1970]; Souriau [1997]; Woodhouse [1992]). In Section II, we propose a definition of physical objects. In Section III, we consider the dynamics of physical objects. In the last Section we summarize the obtained results. Finally, in the appendix we give a brief description of the relevant mathematical structures from symplectic geometry.

\section{Phases of AN ElePhant}

In this section, we propose a definition of physical objects by means of two postulates. It is possible to show that these postulates cannot be implemented in the framework of classical mechanics (see appendix and Catren [2008]). On the contrary, quantum mechanics can be considered a satisfactory implementation of the proposed definition of physical objects.

Physical experience is not a chaotic swarm of disconnected empirical data. As Whitehead put it: 'Sometimes we see an elephant, and sometimes we do not' (Whitehead [1978], p. 4). In other words, physical reality is organized in different objective configurations that can be identified and recognized. According to a standard characterization, an object is a physical configuration that can be completely characterized by specifying the set of the object's objective properties. Such a set will be called the eidos $\varepsilon$ of the physical object. ${ }^{3}$ In order to unpack this characterization, it is necessary to specify what we understand by objective properties. As we shall see, the characterization of objective properties as invariants under a certain set of transformations does not suffice for defining the notion of objective properties. In order to achieve a satisfactory definition of physical objects, it is necessary to take into account that a physical objet does not only have objective

\footnotetext{
${ }^{3}$ This Husserlian terminology is borrowed from (Heelan [2004]).
} 
properties that allow us to identify and recognize it: it also has different phases, aspects or profiles. In general, various kinds of transformations can be performed in order to observe the different phases of an object. For instance, there are objects that exhibit different phases when rotated around a given axis. The transformations that interchange the phases of an object will be called phase transformations of the object. A set of phases connected by means of a given one-parameter family of phase transformations will be called orbit of phases. For instance, the different phases observed when the object is rotated around a given axis belong to the same orbit of phases. Since a phase transformation only modifies the observed phase, the objective properties that define the object are necessarily invariant under phase transformations. In order to stress this fact, phase transformations will also be called automorphisms of the object. In this way, we recover the idea that an object can be defined by means of the invariants under a certain group of transformations (see for example Auyang [1995]; Born [1998]; Nozick [1998]). Following H. Weyl, we can thus state that '[...] objectivity means invariance with respect to the group of automorphisms' (Weyl [1952]). Nevertheless this standard characterization is insufficient for defining objectivity. This problem was clearly stated by $\mathrm{R}$. Nozick [1998]: 'The notion of invariance under transformations cannot (without further supplementation) be a complete criterion of the objectivity of facts, for its application depends upon a selection of which transformations something is to be invariant under.' ${ }^{4}$ The definition of physical objects that we will propose provides this 'further supplementation' by stating that the object's automorphisms are induced by the objective properties of the object. Hence, not only is an objective property invariant under all the object's automorphisms, but it also induces a one-parameter family of automorphisms.

In order to formalize this idea, we will propose a definition of physical objects by means of two fundamental postulates. To do so, we will introduce some terminology. We will say that a physical object realizes a certain number of universal operators in a way that depends on the objet. For example, an object that can be rotated around the $z$-axis realizes (in a particular way that depends on the objet) the universal

\footnotetext{
${ }^{4}$ Analogously, H. Weyl continues the preceding quotation as follows: 'Reality may not always give a clear answer to the question what the actual group of automorphisms is [...]' (Weyl [1952]).
} 
operator that generates universal rotations around the $z$-axis. We will sometimes say that a universal operator makes ingression into the object in a way that depends on the objet. The important point is that two different objects can realize different universal operators or realize differently the same universal operator. Hence, an object can be characterized by the way in which it realizes a particular set of universal operators. Therefore, there are two ways of characterizing an object, namely by means of its objective properties or by specifying how it realizes certain universal operators. Our first postulate unifies these two ways of characterizing an object:

Postulate $\mathbf{\phi}$ : the value of an objective property of a given object specifies the particular way in which the object realizes a universal operator.

The ingression of a universal operator into an object defines what we will call an eigenoperator of the object. For example, there are objects defined (at least partially) by the objective property that specifies how the universal operator that generates universal rotations around the $z$-axis makes ingression into the object. This ingression defines an eigenoperator that generates the object's rotations around the $z$-axis. In this way, postulate $\boldsymbol{\infty}$ states that the particular value $p_{0}$ that an objective property $p$ takes on a given object $\mathcal{O}$ defines an ingression application $\iota_{p_{0}}$ of the form:

$$
\iota_{p_{0}}: \text { universal operator } \xi_{p} \rightarrow \text { eigenoperator } \hat{v}_{p}
$$

Each possible value of the objective property $p$ defines a different ingression of the same universal operator $\xi_{p}$, that is to say a different eigenoperator $\hat{v}_{p}$ of $\mathcal{O}$.

The second postulate of our definition of physical objects specifies the nature of the transformations generated by the object's eigenoperators:

Postulate p: the transformations generated by an object's eigenoperator are phase transformations.

In other words, a transformation generated by one of the object's eigenoperators is not an objective transformation of the object into another object. In the previous example, this means that the object's rotation around the $z$-axis is not an objective transformation of the 
object, but rather an automorphism that leaves the objective properties invariant. These two postulates can be assembled together by stating that an objective property specifies how a universal operator is realized by the object in the form of an eigenoperator that generates automorphisms of the object. We will sometimes summarize this characterization by saying that an objective property induces a oneparameter family of automorphisms. In this way, the object's eidos (i.e. the set of its objective properties) defines the identity of the object by inducing the phase transformations between its different phases. We can therefore propose the following definition:

Definition: An object is a physical configuration that can be completely characterized by specifying the values of the objective properties that induce all the object's automorphisms.

We could say that this definition provides a rigorous formalization of Weyl's prescription: 'Whenever you have to do with a structureendowed entity $\Sigma$ try to determine its group of automorphisms, the group of those element-wise transformations which leave all structural relations undisturbed. You can expect to gain a deep insight into the constitution of $\Sigma$ in this way.' (Weyl [1952]). In the case of the proposed definition of physical objects, the objective properties that define the object do induce the object's automorphisms.

One significant consequence of this definition is that the phase transformations induced by an objective property in the object's eidos cannot modify the other objective properties of the object. Objective properties must therefore be invariant under phase transformations induced by the other objective properties of the same object. Let's consider for example an object defined by the eidos $\varepsilon=\left\{p_{1}, p_{2}, \ldots, p_{n}\right\}$, where each $p_{i}$ is an objective property of the object. This means that it is possible to completely characterize the object by the set of values that the properties $p_{1}, p_{2}, \ldots, p_{n}$ take on the object. The standard definition of objectivity requires that the value of each objective property $p_{i}$ be invariant under a certain group of transformations. Nevertheless - as we have said before - it is not clearly stated which transformations have to be considered. Our definition bypasses this flaw by stating that each objective property $p_{i}$ induces a one-parameter family of automorphisms of the object. Hence, we arrive at the conclusion that each objective property has to be invariant under the automorphisms induced by all 
the other properties in the same eidos. This fact imposes a restrictive condition on the eidos of an object. The eidos is not merely a collection of unrelated objective properties. Each property has to satisfy the condition of being invariant under the phase transformations induced by all the others. If a property $p_{1}$ is invariant under the phase transformations induced by $p_{2}$, we will say that the properties are commensurable or compatible. If a property is modified by the phase transformations induced by an objective property in the object's eidos, we will say that the former is phased out by these phase transformations. Therefore, the eidos is characterized by an internal structure that guarantees the compatibility between the objective properties that define the object. ${ }^{5}$ The object will be completely determined if the eidos contains the maximum number of mutually compatible properties. In particular, if a property $q$ is modified by the phase transformations induced by an objective property $p$ in the object's eidos, then $q$ cannot also be an objective property of the object. This statement can be considered the conceptual translation of the uncertainty principle. In particular, the momentum $p$ is a property that induces transformations in the position $q$ (and vice versa). ${ }^{6}$ Hence, if the momentum $p$ is an objective property in the object's eidos, then the position $q$ cannot also be an objective property. The position $q$ is rather a phase that changes when the object is acted upon by the phase transformations induced by $p$. In other words, since $q$ and $p$ are incompatible, they cannot both be objective properties of the same object. Asking which position is objective in an object with a well-defined momentum $p$ is as nonsensical as looking for the objective (or privileged) side of a die. Nevertheless, even if a die has no objective side, it will show a particular side when thrown. This does not mean that the resulting side was the objective but unknown side of the die, nor that it becomes the objective side of a new die produced by the toss. Analogously, even if a physical system with a well-defined momentum $p$ has no objective position $q$, it will appear in a particular position $q_{1}$ if a measurement of the position is performed.

\footnotetext{
${ }^{5}$ In technical terms, the action induced by a property $g$ on a property $f$ is given by the Poisson bracket $\delta_{g} f=\{f, g\}$. The requirement of internal consistency of the eidos $\varepsilon$ imposes the condition $\{f, g\}=0, \forall f, g \in \varepsilon$. In other words, the eidos is a commutative Poisson algebra.

${ }^{6}$ The Poisson bracket $\{q, p\}=1$ means that the momentum $p$ is the generator of the infinitesimal canonical transformations of the position $q$ (and vice versa).
} 


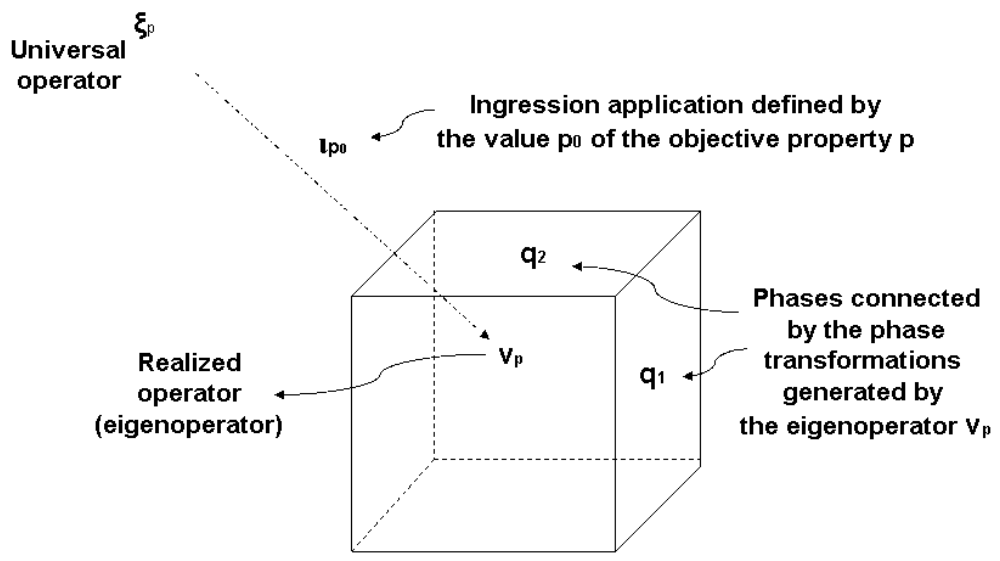

FIG. 1

This does not mean that $q_{1}$ was the objective but unknown position of the system, nor that $q_{1}$ becomes the objective position of a new object produced by the measurement.

Figure 1 resumes the proposed definition of physical objects. The property $p$ is an objective property of the object represented. The value $p_{0}$ of this property specifies how the universal operator $\xi_{p}$ makes ingression into the object. The ingression of the universal operator $\xi_{p}$ defines the eigenoperator $\hat{v}_{p}$. This eigenoperator generates phase transformations that act upon the property $q$. Hence, the different values of this property are just different phases in the orbit of phases generated by the eigenoperator $\hat{v}_{p}$.

According to postulate $\boldsymbol{\beta}$, the transformations induced by the objective properties of an object are phase transformations. As we have shown, the uncertainty principle is a formal consequence of this postulate. If the momentum $p$ is an objective property of an object, then the position $q$ is completely phased out by the phase transformations induced by $p$. Since the classical definition of objective physical states comprises both the exact position and the exact momentum of the system, this postulate cannot be consistently implemented in the framework of classical mechanics. We can also argue differently. If both the position and the momentum were included in the object's eidos, then both the position and the momentum would be phased out by 
the phase transformations induced by the momentum and the position respectively. Therefore, both the position and the momentum would only be non-objective phases, and the physical system would have no objective properties at all. We can thus conclude that the classical definition of states by means of both $q$ and $p$ is incompatible with our definition of physical objects. The classical definition of a physical state is consistent only if we deny that the transformations induced by an objective property of the system are phase transformations. In fact, in classical mechanics the transformations induced by an objective property are not interpreted as phase transformations, but rather as transformations between states that are objectively different. For example, the transformations induced by the Hamiltonian are interpreted as temporal evolutions between different objective states. In this way, the definition of classical states becomes consistent. Nevertheless, objective properties can no longer be defined as quantities that induce the object's automorphisms. Hence, the classical definition of both objective properties and physical objects remains problematic. The situation has thus been conveniently reversed: the problem is no longer how to recover objectivity in quantum mechanics, but rather how to define classical objects in a consistent manner.

For the sake of simplicity we have only considered the case of an object with a well-defined momentum and a completely undetermined position. The reciprocal case - a well-defined position with an undetermined momentum - is completely analogous. In the general case, both the position and the momentum are subject to certain indeterminacies. In fact, the flexibility of quantum mechanics' formalism makes it possible to define physical objects characterized by properties which are neither objective properties nor phases, but rather a mixture of both. In these cases, neither $q$ nor $p$ are sharp objective properties of the object. For example, if $q$ is an unsharp objective property of an object, then the induced phase transformations are unsharp phase transformations. Therefore, the conjugated momentum $p$ is phased out only partially. Hence, $p$ is in turn an unsharp objective property that partially phases out the coordinate $q$. Therefore, $q$ cannot be a sharp objective property (as it was assumed at the beginning) and the circle closes consistently. This means that a certain property can be partially considered an unsharp objective property of the object and partially a 
phase. It follows that the mere distinction between objective properties and phases does not suffice for treating generic cases. The resulting subtle equilibrium between unsharp objective properties and unsharp non-objective phases is formally governed by the uncertainty principle.

\section{The Revenge of Zeno}

"[...] comment l'objet qui se meut serait-il en un point de son trajet?"

H. Bergson, La pensée et le mouvant (Bergson [1938, p. 158])

The analysis presented in the previous section makes no reference to temporal processes. Since physics, as it is usually understood, studies the temporal evolution of physical systems, we will now introduce a temporal parameter $t$. The consideration of temporal processes allows to shift the discussion from momenta $p_{i}$ (observables that generate infinitesimal canonical transformations of positions $q^{i}$ ) to velocities $\dot{q}^{i}$ (infinitesimal temporal variations of $q^{i}$ ).

We will begin by noting that an object $\mathcal{O}$ characterized by a welldefined velocity lacks, by definition, a well-defined position. Analogously, a nomad is a person characterized by the property of not having a well-defined position. We claim that this trivial fact contains the conceptual kernel of the uncertainty principle for positions and velocities. One might argue that this lack of a well-defined position can be bypassed by decomposing the movement in instantaneous objects $\mathcal{O}_{t}$ that evolve in time, that is to say that change objectively as time passes. Even though the state of motion of the object $\mathcal{O}$ makes it impossible to assign it a constant position, it might still be possible to define the objective positions of the different instantaneous objects $\mathcal{O}_{t}$. According to the standard terminology, the objects $\mathcal{O}_{t}$ might be called the instantaneous states of the object $\mathcal{O}$. We will now analyze whether this strategy can be consistently pursued in the framework of our definition of physical objects.

In what follows, we will restrict the analysis to the simplest case. Let's consider an object $\mathcal{O}$ consisting of a free particle moving with a constant momentum $p$ (or a constant velocity $\dot{q}$ ). In principle, we could decompose $\mathcal{O}$ in a sequence of instantaneous objects $\mathcal{O}_{t}$. Each of 
these instantaneous objects $\mathcal{O}_{t}$ would be characterized by the objective property $p$ (which induces the displacements in $q$ ) and its position $q(t)$ at $t$. In other words, as in classical mechanics, both $p$ and $q(t)$ would be objective properties of the instantaneous object $\mathcal{O}_{t}$. Even if $q(t)$ is not an objective property of $\mathcal{O}$, it might still be considered an objective property of the instantaneous object $\mathcal{O}_{t}$. Nevertheless, this decomposition of $\mathcal{O}$ in instantaneous objects $\mathcal{O}_{t}$ characterized by both $p$ and $q(t)$ is inconsistent with the proposed definition of physical objects. Since the position $q(t)$ changes in time, the different instantaneous objects $\mathcal{O}_{t}$ are objectively different. This results from the fact that the position $q(t)$ is considered an objective property of $\mathcal{O}_{t}$. Hence, the different instantaneous objects $\mathcal{O}_{t}$ differ in the objective property $q(t)$. This means that temporal evolution is a non trivial objective modification of the instantaneous objects $\mathcal{O}_{t}$. Therefore, the Hamiltonian $h$, which induces the transformations of $t$, cannot be an objective property of the instantaneous object $\mathcal{O}_{t}$. According to our definition of physical objects, if $h$ were an objective property of $\mathcal{O}_{t}$, then the transformations induced by $h$ would be phase transformations that could not objectively modify the object. Since temporal evolution objectively modifies the instantaneous objects $\mathcal{O}_{t}$, the Hamiltonian $h$ cannot be an objective property of $\mathcal{O}_{t}$. Nevertheless, this conclusion contradicts the fact that if $p$ is an objective property of $\mathcal{O}_{t}$, then $h=\frac{p}{2 m}$ should also be an objective property of $\mathcal{O}_{t}$. We can also argue in the opposite sense. Since $p$ is an objective property of $\mathcal{O}_{t}$, the Hamiltonian $h=\frac{p}{2 m}$ is also an objective property of $\mathcal{O}_{t}$. Hence, according to our definition of physical objects, the transformations induced by $h$ are phase transformations. ${ }^{7}$ Therefore, the different $\mathcal{O}_{t}$ are not different instantaneous objects $\mathcal{O}_{t}$, but rather different phases of the same object $\mathcal{O}$. We can thus conclude that the object $\mathcal{O}$ cannot be consistently decomposed in different instantaneous objects $\mathcal{O}_{t}$. Hence, the object $\mathcal{O}$ is an indecomposable object with different non-objective temporal phases. In other words, what we observe at different times are not different instantaneous objects $\mathcal{O}_{t}$, but rather different non-objective temporal phases of the same object $\mathcal{O}$.

\footnotetext{
${ }^{7}$ This statement is a rigorous interpretation of the fact that '[...] the motion of a mechanical system corresponds to the continuous evolution or unfolding of a canonical transformation.' (Goldstein [1981]).
} 
These considerations do not mean that it is impossible to define physical objects that change objectively in time. We simply claim that a system moving with a constant velocity cannot be analyzed in terms of instantaneous objects (or objective states) that change objectively in time. However, in principle, it is possible to define an instantaneous object $\mathcal{O}_{t}$ such that its eidos contains the property $t .{ }^{8}$ Since the time $t$ is an objective property of the instantaneous object $\mathcal{O}_{t}$, the property $h$ is phased out by the phase transformations induced by $t$. Hence, the transformations induced by $h$ are no longer phase transformations, but rather objective transformations of the object. Therefore, at different times $t$ and $t^{\prime}$, there are instantaneous objects $\mathcal{O}_{t}$ and $\mathcal{O}_{t^{\prime}}$ that are objectively different. ${ }^{9}$

\section{CONClusion}

We have defined a physical object as a set of mutually compatible objective properties such that each objective property induces a one-parameter family of automorphisms. The compatibility condition guarantees that the objective properties are invariant under the automorphisms induced by all the other objective properties of the same object. The uncertainty principle is a direct consequence of the mutual imbrication between objective properties and non-objective phases: if $p$ is a sharp objective property of an object, then the property $q$ (phased out by the phase transformations induced by $p$ ) cannot also be an objective property.

We could restate Einstein's requirement by saying that a satisfactory physical theory has to provide a complete objective description of physical reality (Einstein et al. [1935]). Firstly, this means that every objective property of physical reality should have a counterpart in the theory. Secondly, non-objective properties should not be mistaken for objective properties by the theory. The classical description of a physical system includes both its objective properties and its

\footnotetext{
${ }^{8}$ We are supposing that it is possible to treat time and the Hamiltonian as another pair of conjugated canonical variables. In fact, this is possible in the framework of the so-called parameterized systems (see for example Lanczos [1986] and Castagnino et al. [2002]).

${ }^{9}$ The arguments presented in this section suggest that a satisfactory comprehension of the uncertainty principle for time and energy might be an essential component of a consistent interpretation of quantum mechanics.
} 
non-objective phases. Unlike classical mechanics, quantum mechanics provides a complete objective description of physical systems.

\section{APPENDIX}

We will now give a brief account of the formal structures that underlie the proposed definition of physical objects (for more details see Abraham and Marsden [1978]; Catren [2008]; Libermann and Marle [1987]; Marsden and Ratiu [1999]). A symplectic action of a Lie group $G$ (of Lie algebra $\mathrm{g}$ ) on a symplectic manifold $(M, \omega)$ is a group action $\Phi: G \times M \rightarrow M$ that preserves the symplectic form $\omega$, i.e. that satisfies $\Phi_{g}^{*} \omega=\omega$, where $\Phi_{g}^{*}$ is the pullback defined by the map $\Phi_{g}(\cdot) \doteq \Phi(g, \cdot)$. Such an action defines a map $\iota: \mathrm{g} \rightarrow T M$ (that we have called ingression) between Lie algebra elements $\xi \in \mathrm{g}$ (that we have called universal operators) and fundamental vector fields $v_{\xi}$ on $M$ (that we have called realized operators). The fundamental vector field $v_{\xi}$ is given by the expression

$$
v_{\xi}(x)=\left.\frac{d}{d \lambda}(\exp (-\lambda \xi) \cdot x)\right|_{\lambda=0},
$$

where $x \in M$ and $\xi \in \mathrm{g}$. The symplectic action is said to be Hamiltonian if the ingression map $\iota: \mathrm{g} \rightarrow T M$ can be "factorized" as follows

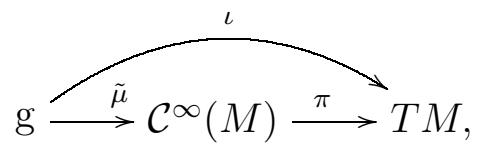

where $\tilde{\mu}: \mathrm{g} \rightarrow \mathcal{C}^{\infty}(M)$ is the so-called co-momentum map and $\pi$ : $\mathcal{C}^{\infty}(M) \rightarrow T M$ is the map between classical observables on $M$ and the so-called Hamiltonian vector fields. A classical observable $f \in \mathcal{C}^{\infty}(M)$ defines a Hamiltonian vector field $v_{f}$ by means of the expression $i_{v_{f}} \omega=$ $d f$, where $i_{v_{f}} \omega$ denotes the contraction of $v_{f}$ with the symplectic 2-form $\omega$. The Hamiltonian vector field $v_{f}$ is the generator of the symplectic diffeomorphisms $\phi_{\lambda}^{f}: M \rightarrow M$, that is to say of the canonical transformations induced by the observable $f$. In $\mathbb{R}^{2}$, the Hamiltonian vector field associated to an observable $f \in \mathcal{C}^{\infty}(M)$ is given by the expression

$$
v_{f}=\frac{\partial f}{\partial p} \frac{\partial}{\partial q}-\frac{\partial f}{\partial q} \frac{\partial}{\partial p} .
$$

In other words, a symplectic action is Hamiltonian if the fundamental vector field $v_{\xi}$ that realizes on $M$ the universal operator $\xi \in$ g can also be obtained as the Hamiltonian vector field $v_{f}$ associated to a physical observable $f$. It might seem that we have all the elements for 
implementing the proposed definition of physical objects. In fact, the sequence of maps (2) seems to be the formal implementation of the application (1) between universal operators and eigenoperators. According to (2), the objective properties $f \in \mathcal{C}^{\infty}(M)$ "factorize" the ingression on $M$ of universal operators $\xi \in$ g. Nevertheless, the two postulates that we used for defining physical objects are not satisfied in the classical framework. The main problem is that the homomorphism $\pi$ between the Poisson algebra of classical observables $f \in \mathcal{C}^{\infty}(M)$ and the Lie algebra of classical operators $v_{f}$ (under the Lie bracket of vector fields) is not an isomorphism of Lie algebras. This is a consequence of the fact that the map $f \mapsto v_{f}$ is not injective (since $v_{k}=0$ for any $k \in \mathbb{R})$. The fundamental consequence of this non-injectivity is that

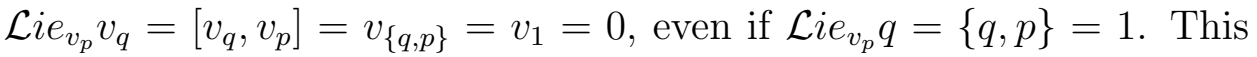
means that in classical mechanics, a non-trivial transformation (generated by the classical operator $v_{p}$ ) of the value of an objective property $q$ does not necessarily modify the realized operator $v_{q}$. This means that in classical mechanics, different states (characterized by different values of $q$ ) do not realize differently the same universal operator in $g$. Hence, an objective property cannot be defined - as we did in postulate - as a quantity that specifies the particular way in which the object realizes a universal operator. As for postulate $\mathbf{s}$ (according to which a realized operator generates automorphisms of the object), we have already shown why it cannot be consistently implemented in classical mechanics. In order to satisfy these postulates, it is necessary to extend the classical operators $v_{f}$ to quantum operators $\hat{v}_{f}$ such that the latter satisfy Dirac's quantization conditions. In the framework of geometric quantization, this can be done by means of the so-called prequantization formalism (Brylinski [1993]; Kostant [1970]; Souriau [1997]; Woodhouse [1992]).

\section{REFERENCES}

[1] Abraham, R. and Marsden, J.E. [1978]: Foundations of Mechanics, second ed., Cambridge, MA: Addison-Wesley Publishing Company.

[2] Auyang, S.Y. [1995]: How is Quantum Field Theory Possible?, Oxford-New York: Oxford University Press.

[3] Bergson, H. [1938]: La pensée et le mouvant, Paris, Presses Universitaires de France. 
[4] Born, M. [1998]: 'Physical Reality', in E. Castellani (ed.), 1998, Interpreting Bodies. Classical and Quantum Objects in Modern Physics, New Jersey: Princeton University Press.

[5] Brylinski, J.-L. [1993]: Loop Spaces, Characteristic Classes, and Geometric Quantization, Progr. Math. 107, Boston MA: Birkhäuser Boston Inc.

[6] Catren, G. [2008]: 'On Classical and Quantum Objectivity', Foundations of Physics, 38, pp. 470-487.

[7] Castagnino, M., Catren, G. and Ferraro, R. [2002]: 'Time Asymmetries in Quantum Cosmology and Boundary Conditions to the Wheeler-DeWitt Equation', Class. Quant. Grav. 19, No. 18, pp. 4729-4746.

[8] Einstein, A., Podolsky, B. and Rosen, N. [1935]: 'Can Quantum-Mechanical Description of Physical Reality be Considered Complete?', Phys. Rev. 47, pp. 777-780.

[9] Goldstein, H. [1981]: Classical Mechanics, Cambridge, MA: Addison-Wesley Publishing Company.

[10] Heelan, P. [2004]: 'The Phenomenological Role of Consciousness in Measurement', Mind and Matter, Vol. 2(1), pp. 61-84.

[11] Hilgevoord, J. and Uffink, J. [2006]: 'The Uncertainty Principle', in E. N. Zalta (ed.), The Stanford Encyclopedia of Philosophy.

[12] Kostant, B. [1970]: 'Quantization and Unitary representations', Lecture Notes in Mathematics, Vol. 170, Berlin-Heidelberg-New York: Springer-Verlag.

[13] Lanczos, C. [1986]: The Variational Principles of Mechanics, New York: Dover.

[14] Libermann, P. and Marle, C.-M. [1987]: Symplectic Geometry and Analytical Mechanics, Dordrecht, Holland: D. Reidel Publishing Company.

[15] Marsden, J.E. and Ratiu, T.S. [1999]: Introduction to Mechanics and Symmetry, second ed., New York: Springer-Verlag.

[16] Mittelsteadt, P. [2006]: 'Einstein's Objections against Quantum Mechanics', Physics and Philosophy, http://hdl.handle.net/2003/22995.

[17] Nozick, R. [1998]: 'Invariance and Objectivity', Proceedings and Addresses of the American Philosophical Association, Vol. 72, No. 2, pp. 21-48.

[18] Souriau, J.-M. [1997]: Structure of Dynamical Systems. A Symplectic View of Physics, Cambridge, MA: Birkhäuser Boston.

[19] Weyl, H. [1952]: Symmetry, Princeton: Princeton University Press.

[20] Whitehead, A.N. [1978]: Process and Reality, New York: The Free Press.

[21] Woodhouse, N. [1992]: Geometric Quantization, second ed., Oxford: Oxford University Press. 